\title{
Prüfer Domain and Affine Scheme
}

\author{
Koji SEKIGUCHI
}

\author{
Sophia University
}

\section{Introduction.}

Valuation theory has an intimate relation with number theory and algebraic geometry. The following Theorem 0 , for instance, shows a role of discrete valuation rings.

Let $K$ be a field. We consider the following four conditions for a set $W$ consisting of valuation rings with quotient field $K$ :

(W-0) $\quad W \neq \varnothing$.

(W-1) If $R \in W$, then $R$ is a discrete valuation ring.

(W-2) For any $x \in K$, the set $\{R \in W \mid x \notin R\}$ is finite.

(W-3) If $R_{1}, R_{2} \in W, U^{(1)} R_{1} \cap m\left(R_{2}\right) \cap \cap_{R \in W} R=\varnothing$, then $R_{1}=R_{2}$, where $m(R)$ is the unique maximal ideal of a local ring $R$, and $U^{(i)} R=$ $1+m(R)^{i}(i \geqq 1)$. Then,

THEOREM 0. Let $K$ be a field. Then there exists an inclusionreversing bijection between the set of all Dedekind domains $A$ with quotient field $K$, and the set of all $W$ satisfying the conditions (W-0), $(W-1),(W-2)$ and $(W-3)$. The bijection is defined by

$$
\left\{\begin{aligned}
A \longmapsto & W: \begin{array}{l}
W \text { is the set of all } P \text {-adic valuation rings } \\
\text { defined by the maximal ideals } P \text { of } A,
\end{array} \\
W \longmapsto A: & A \text { is the intersection of all valuation rings } \\
& \text { belonging to } W .
\end{aligned}\right.
$$

For a proof, see [3], Theorem 1.3, Theorem 1.4, and p. 441.

In this paper, we shall generalize Theorem 0 (see Theorems 9 and 13) 
and prove the analogous result (see Theorem 15). And as applications of these results, we shall show Theorems 20, 21, 22, 23, 25 and 28. Especially, Theorem 15 (together with Theorem 0) implies that the conditions (W-0), (W-1), (W-2) and (W-3) characterize the set of all closed points of a noetherian affine scheme $(\neq \varnothing, \neq\{K\})$ consisting of valuation rings of $K$. And Theorem 22 is a characterization of global fields of dimension zero or one, without using the Archimedean valuations. Comparing with this, Artin-Whaples' theory is a characterization of global fields of dimension one, using the Archimedean valuations (see [3], p. 470).

The author wishes to express his thanks to Professor Yukiyosi Kawada.

$\S 1$. Here we induce a topology and a presheaf of rings on a set consisting of valuation rings. Let $K$ be a field. We denote by Zar $K$ the set of all valuation rings with quotient field $K$. For a subset $E$ of $K$, we put $\operatorname{Zar}(K \mid E)=\{R \in \operatorname{Zar} K \mid E \subset R\}$. Let $\left(E_{i}\right)_{i \in I}$ be a family of subsets of $K$. Then:

$$
\begin{aligned}
& \operatorname{Zar}\left(K \mid \bigcup_{i \in I} E_{i}\right)=\bigcap_{i \in I} \operatorname{Zar}\left(K \mid E_{i}\right), \\
& \operatorname{Zar}\left(K \mid \bigcap_{i \in I} E_{i}\right) \supset \bigcup_{i \in I} \operatorname{Zar}\left(K \mid E_{i}\right) .
\end{aligned}
$$

For $R_{1}, R_{2} \in \operatorname{Zar} K$, we have

$$
R_{1} \subset R_{2} \text { if and only if } m\left(R_{2}\right) \subset m\left(R_{1}\right),
$$

and then we also have,

$$
m\left(R_{2}\right) \in \operatorname{Spec} R_{1}, \quad R_{2}=\left(R_{1}\right)_{m\left(R_{2}\right)}, \quad R_{1} / m\left(R_{2}\right) \in \operatorname{Zar}\left(R_{2} / m\left(R_{2}\right)\right) .
$$

Topology. Letting $I$ be a finite set in (1), there exists a unique topology of Zar $K$ with open basis

$$
\Sigma=\{\operatorname{Zar}(K \mid E) \mid E \text { is a finite subset of } K\} .
$$

We call an element of $\Sigma$ a fundamental open set of Zar $K$. Until the end of this paper, we always induce the relative topology of the topology defined by (5), on a subset $X$ of Zar $K$. It is called the Zariski topology on $X$. Then for $R \in X$, we have

$$
\overline{\{R\}}=\left\{R^{\prime} \in X \mid R^{\prime} \subset R\right\} .
$$

Hence $X$ is a $T_{0}$-space. Especially, if $A$ is a subring of $K$ and $X=$ $\operatorname{Zar}(K \mid A)$, then the mapping defined by 


$$
\begin{gathered}
\overline{\{R\}} \longrightarrow \operatorname{Zar}(R / m(R) \mid A+m(R) / m(R)) \\
\stackrel{\omega}{W} \\
R^{\prime} \longmapsto R^{\prime} / m(R)
\end{gathered}
$$

is an inclusion-preserving homeomorphism. And if $\varphi: R \rightarrow R / m(R)$ is the canonical map, then the mapping

$$
Q \longmapsto \varphi^{-1}(Q)
$$

is the inverse map of (7).

Presheaf of rings. Let us induce the presheaf $\mathcal{O}_{X}$ on a subset $X$ of Zar $K$. For an open set $U$ of $X$, we put

$$
\mathscr{O}_{X}(U)=\left\{\begin{array}{cl}
\cap_{R \in U} R, & \text { where } U \neq \varnothing . \\
0, & \text { where } U=\varnothing .
\end{array}\right.
$$

Let $U, V$ be two open subsets of $X$ and $U \subset V$. If $U \neq \varnothing$, then $\mathscr{O}_{X}(V) \subset$ $\mathcal{O}_{X}(U) \subset K$ and we denote by $\rho_{V, U}$ the inclusion map. If $U=\varnothing$, we put $\rho_{V, U}=0$-map. Then it is clear that $\mathcal{O}_{X}$ is a presheaf of rings on $X$. And for a family $\left(V_{i}\right)_{i \in I}$ of non empty open subsets of $X$, we have

$$
\mathscr{O}_{X}\left(\cup_{i \in I} V_{i}\right)=\bigcap_{i \in I} \mathcal{O}_{X}\left(V_{i}\right)
$$

REMARK. In general, for a subset $W$ of Zar $K$, we put

$$
\text { If } W=\varnothing \text {, then } \bigcap_{R \in W} R=K \text { and } \mathcal{O}_{W}(W)=0 \text {. }
$$

Lemma 1. Let $K$ be a field and $X$ a subset of Zar $K$. Then:

(i) the presheaf $\mathcal{O}_{X}$ defined by (8) satisfies the locally uniqueness conditions.

(ii) for any $R \in X$, we have $\mathscr{O}_{X, R} \stackrel{\sim}{\rightarrow} R$.

(iii) the presheaf $\mathscr{O}_{X}$ satisfies the locally existence conditions if and only if $X$ is either irreducible or empty.

Proof. (i) is obvious.

(ii) By the definition of $\rho_{V, U}$, we may assume $\mathscr{O}_{X, R}=\cup_{U} \mathcal{O}_{X}(U)$, for all $R \in X$, where $U$ runs over the set of all open subsets of $X$ containing $R$. Then let us prove $R=\mathscr{O}_{X, R}$. It is clear that $R \supset \mathcal{O}_{X, R}$. Conversely, for any $x \in R, U=X \cap \operatorname{Zar}(K \mid\{x\})$ is an open neighborhood of $R$ in $X$. For any $R^{\prime} \in U$, we have $x \in \mathcal{O}_{X}(U)$, since $x \in R^{\prime}$. Thus we obtain $R \subset \mathcal{O}_{X, R}$.

(iii) Since $X=\varnothing=\operatorname{Spec} 0$ is an affine scheme, we may assume $X \neq \varnothing$. Proof of $\Longleftarrow$. Let $U=\cup_{i \in I} V_{i}$ be an open covering of $U$, and $s_{i} \in \mathscr{O}_{X}\left(V_{i}\right)$ satisfy the conditions $\rho_{V_{i}, V_{i} \cap V_{j}}\left(s_{i}\right)=\rho_{V_{j}, V_{i} \cap V_{j}}\left(s_{j}\right)$ for all $i, j \in I$. We may 
assume $V_{i} \neq \varnothing$ for any $i \in I$. Since $X$ is irreducible, we have $V_{i} \cap V_{j} \neq \varnothing$. Hence $s_{i}=s_{j}$ in $K$. Let $s=s_{i} \in K$ for all $i \in I$. Then we obtain $s \in \mathcal{O}_{X}(U)$ by (9). It is clear that $\rho_{U, V_{i}}(s)=s_{i}$. Proof of $\Rightarrow$. Let us assume that $X$ is reducible. Then there exist two open subsets $V, W$ of $X$ such that $V, W \neq \varnothing$ and $V \cap W=\varnothing$, since $X$ is not empty. If we put $U=V \cup W$, $s_{1}=0 \in \mathscr{O}_{X}(V), s_{2}=1 \in \mathcal{O}_{X}(W)$, then it is impossible that there exists an element $s \in \mathcal{O}_{X}(U)$ such that $\rho_{U, V}(s)=s_{1}$ and $\rho_{U, W}(s)=s_{2}$.

Corollary. Let $X$ be an irreducible subset of $\operatorname{Zar} K$. Then $\left(X, \mathcal{O}_{X}\right)$ is a locally ringed space.

REMARK. In general, the homeomorphisms (7) and (7') are not isomorphisms of locally ringed spaces. Let $Y=\overline{\{R\}} \subset X=\operatorname{Zar}(K \mid A)$, and we renew the definition of sheaf on $Y$ by

$$
\mathcal{O}_{Y}^{\prime}(U)=\bigcap_{R^{\prime} \in U} R^{\prime} / m(R)
$$

for a non empty open subset $U$ of $Y$. Then the mappings (7) and ( $\left.7^{\prime}\right)$ become isomorphisms of locally ringed spaces. However, until the end of this paper, we always induce the presheaf $\mathcal{O}_{X}$ of rings defined by (8) on a subset $X$ of Zar $K$.

Lемма 2. Let $K$ be a field and $X$ a non empty subset of Zar $K$. Then $\operatorname{Zar}\left(K \mid \mathcal{O}_{X}(X)\right)$ is the intersection of all fundamental open sets of Zar $K$ containing $X$.

Proof. Let $\Sigma_{x}$ be the set of all fundamental open sets of Zar $K$ containing $X$. Then we have $\Sigma_{X}=\{\operatorname{Zar}(K \mid E) \mid E$ is a finite subset of $\left.\mathcal{O}_{X}(X)\right\}$. Hence, by (1), we obtain $\operatorname{Zar}\left(K \mid \mathcal{O}_{X}(X)\right)=\cap_{E} \operatorname{Zar}(K \mid E)=\cap_{U \in \Sigma_{X}} U$. Q.E.D.

$\S 2$. Here we consider the integral domains which are integrally closed in a fixed field, as a preparation of $\S 3$. First, we shall admit the following result called Chevalley's lemma from valuation theory.

Lemma 3. Let $K$ be a field, $A$ a subring of $K$ and $F$ an algebraically closed field. Then for any ring homomorphism $\varphi: A \rightarrow F$, there exist $R \in \operatorname{Zar}(K \mid A)$ and ring homomorphism $\psi: R \rightarrow F$ such that $\varphi=\left.\psi\right|_{A}$ (restriction).

The following two lemmas are proved by Lemma 3.

Lemma 4. Let $K$ be a field and $A$ a subring of $K$. Then the mapping $\Phi_{K \mid A}$ defined by 


$$
\begin{array}{cc}
\operatorname{Zar}(K \mid A) & \longrightarrow \operatorname{Spec} A \\
\Phi_{K \mid A}: & \mathscr{U} \\
R & \longmapsto A \cap m(R)
\end{array}
$$

is surjective and continuous.

CoRollary. $\operatorname{dim} \operatorname{Zar}(K \mid A) \geqq \operatorname{dim} A+\operatorname{tr}-\operatorname{deg}_{Q A} K$, where $Q A$ denotes the quotient field of $A$. p. 5.

For the definition of the dimension of topological spaces, see [2],

Lemma 5. Let $K$ be a field and $A$ a subring of $K$. Then:

(i) the integral closure of $A$ in $K$ is $\cap_{R \in \operatorname{Zar}(K \mid A)} R$.

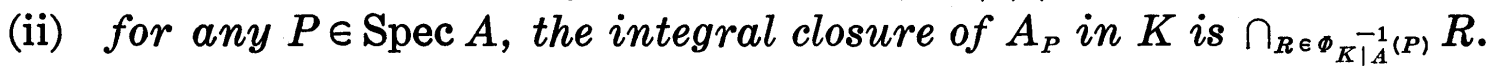

Morphism $\Phi_{K \mid A}$ of sheaves of rings. Let $K$ be a field, $A$ a subring of $K, X=\operatorname{Zar}(K \mid A)$ and $\tilde{A}$ the structure sheaf of affine scheme Spec $A$. From (6), we see that $X$ is irreducible. Then the morphism $\Phi_{K \mid A}: \widetilde{A} \rightarrow$ $\left(\Phi_{K \mid A}\right)_{*} \mathcal{O}_{X}$ of sheaves of rings on Spec $A$ is defined by

$$
\begin{gathered}
\tilde{A}(U) \longrightarrow \Phi_{K \mid A}^{*}(U): \quad \overbrace{X}\left(\Phi_{K \mid A}^{-1}(U)\right) \\
\bigcap_{P \in U} A_{P} \smile \bigcap_{P \in U^{\prime}}\left(\text { the integral closure of } A_{P} \text { in } K\right)
\end{gathered}
$$

for a non empty open subset $U$ of $\operatorname{Spec} A$. And the induced map of $\Phi_{K \mid A}$ on the stalks is denoted by

$$
\left(\Phi_{K \mid A}^{\#}\right)_{R}: A_{P} \smile R
$$

for $R \in X$ and $P=\Phi_{K \mid A}(R) \in \operatorname{Spec} A$. Since (12) is a local homomorphism,

$$
\left(\Phi_{K \mid A}, \Phi_{K \mid A}\right):\left(X, \circlearrowleft_{X}\right) \longrightarrow(\operatorname{Spec} A, \widetilde{A})
$$

is a morphism of locally ringed spaces. By (11), we obtain that $A$ is integrally closed in $K$ if and only if $\Phi_{K \mid A}: \widetilde{A} \rightarrow\left(\Phi_{K \mid A}\right)_{*} \mathcal{O}_{X}$ is an isomorphism. Especially, if $U=D(f)=\{P \in \operatorname{Spec} A \mid f \notin P\} \quad(f \in A, f \neq 0)$, then we have $\Phi_{K \mid A}^{-1}(D(f))=\operatorname{Zar}\left(K \mid A_{f}\right)$ and

$$
\begin{aligned}
\Phi_{K \mid A}(D(f)): & A_{f} \rightleftharpoons \mathcal{O}_{X}\left(\operatorname{Zar}\left(K \mid A_{f}\right)\right): \text { the } \\
& \text { integral closure of } A_{f} \text { in } K .
\end{aligned}
$$

LEMMA 6. Let $K$ be a field and $A$ a subring of $K$.

(i) The next four conditions are equivalent:

(a) $\operatorname{Zar}(K \mid A)=\{K\}$. 
(b) $\operatorname{dim} \operatorname{Zar}(K \mid A)=0$.

(c) $K$ is an integral extension over $A$.

(d) $A$ is a field and $K$ is an algebraic extension over $A$.

(ii) If $\operatorname{dim} \operatorname{Zar}(K \mid A) \leqq 1$, then the equality $\operatorname{dim} \operatorname{Zar}(K \mid A)=\operatorname{dim} A+$ tr-deg ${ }_{Q A} K$ holds.

Proof. (i) is obvious from Lemma 5.

(ii) is obvious from the corollary of Lemma 4 and (i).

Q.E.D.

COROLlaRY. Zar $K=\{K\}$ if and only if $K$ is an algebraic extension over a finite field.

Lemma 7. Let $K$ be a field, $A$ a subring of $K$ and $R \in \operatorname{Zar}(K \mid A)$. Then the following three conditions are equivalent:

(a) $R$ is a closed point of $\operatorname{Zar}(K \mid A)$.

(b) $R / m(R)$ is an integral extension over $A+m(R) / m(R)$.

(c) $\Phi_{K \mid A}(R) \in m$-Spec $A$ (hence $A+m(R) / m(R)$ is a field) and $R / m(R)$ is an algebraic extension over $A+m(R) / m(R)$, where $m$-Spec $A$ denotes the set of all maximal ideals of $A$.

Proof. Obvious from (6), (7) and Lemma 6.

Q.E.D.

Proposition 8. Let $K$ be a field, $A$ a subring of $K, X=\operatorname{Zar}(K \mid A)$ and $W$ the set of all closed points of $X$. Then:

(i) $\Phi_{K \mid A}(W)=m$-Spec $A$. Hence by restriction, the mapping $\Phi_{K \mid A}: W \rightarrow$ $m$-Spec $A$ is well-defined and surjective.

(ii) for any $R \in X$, there exists $R_{0} \in W$ such that $R_{0} \subset R$. Therefore, we also have $W \neq \varnothing$ and $\mathcal{O}_{W}(W)=\mathcal{O}_{X}(X)$.

Proof. (i) It is clear that $\Phi_{K \mid A}(W) \subset m$-Spec $A$ by Lemma 7. Conversely, let $P \in m$-Spec $A, F$ the algebraic closure of $A / P$ and $\phi: A \rightarrow$ $A / P \subset F$ the canonical mapping. Then by Lemma 3, we have $R \in \operatorname{Zar}(K \mid A)$ and ring homomorphism $\psi: R \rightarrow F$ such that $\left.\psi\right|_{A}=\varphi$. By Lemma 7, we obtain $P=\Phi_{K \mid A}(R)$ and $R \in W$.

(ii) For any $R \in X$, there exists a closed point $Q_{0}$ of $\operatorname{Zar}(R / m(R)$ $A+m(R) / m(R))$. Let $R_{0}$ be the closed point of $\overline{\{R\}}$ corresponding to $Q_{0}$ by the mapping (7). Then we have $R_{0} \in W$ and $R_{0} \subset R$. Q.E.D.

REMARK. In general, the equality $W=\Phi_{K \mid A}^{-1}(m$-spec $A)$ does not hold. Zar $K$ :

Here we consider the following two conditions for a subset $W$ of

(W-4) If $\cap_{R \in W} R \subset R^{\prime} \in \operatorname{Zar} K$, then there exists $R_{0} \in W$ such that $R_{0} \subset R^{\prime}$. 
(W-5) If $R_{1}, R_{2} \in W, R_{1} \subset R_{2}$, then $R_{1}=R_{2}$.

It is clear by (2) that (W-4) is equivalent to the following condition:

$$
\operatorname{Zar}\left(K \mid \cap_{R \in W} R\right)=\cup_{R \in W} \operatorname{Zar}(K \mid R) .
$$

And $W$ satisfies (W-5) if and only if $W$ is a $T_{1}$-space. Moreover, we observe that (W-4) implies (W-0) by ( $\left.8^{\prime}\right)$.

Next we consider the relation between $X, A$ and $W$. Let us fix a field $K$, and we put

$$
\begin{array}{r}
\tilde{\mathscr{Q}}=\{X \subset \operatorname{Zar} K \mid X \text { is expressed as an intersection of } \\
\text { some fundamental open sets of } \operatorname{Zar} K\},
\end{array}
$$

$$
\begin{aligned}
& \tilde{\mathscr{A}}=\{A \mid A \text { is a subring of } K \text { and is integrally closed in } K\}, \\
& \tilde{\mathscr{W}}=\{W \subset \operatorname{Zar} K \mid W \text { satisfies (W-4) and (W-5) }\} .
\end{aligned}
$$

Then we define the mappings between $\tilde{\mathscr{X}}, \tilde{\mathscr{A}}$ and $\tilde{\mathscr{W}}$ by

$$
\begin{aligned}
& X \longmapsto A=\mathcal{O}_{X}(X), \quad A \longmapsto X=\operatorname{Zar}(K \mid A), \\
& \left\{\begin{array}{l}
A \longmapsto W=\{R \in \operatorname{Zar}(K \mid A) \mid R / m(R) \text { is an integral } \\
\text { extension over } A+m(R) / m(R)\}, \\
W \longmapsto A=\mathcal{O}_{W}(W),
\end{array}\right. \\
& \left\{\begin{array}{l}
W \longmapsto X=\{R \in \operatorname{Zar} K \mid \overline{\{R\}} \cap W \neq \varnothing\}, \\
X \longmapsto W: \text { the set of all closed points of } X .
\end{array}\right.
\end{aligned}
$$

REMARK. Instead of (19), we can also write

$$
\begin{aligned}
W \longmapsto X & =\underset{R \in W}{\cup} \operatorname{Zar}(K \mid R)=\operatorname{Zar}\left(\left.K\right|_{R \in W} R\right) \\
& =\left\{R_{P} \mid R \in W, P \in \operatorname{Spec} R\right\} .
\end{aligned}
$$

Then,

THeOREM 9. Let $K, \tilde{\mathscr{X}}, \tilde{\mathscr{A}}, \tilde{\mathscr{W}}$ be as above. Then the mappings (17), (18) and (19) between $\tilde{\mathscr{Q}}, \tilde{\mathscr{A}}$ and $\tilde{\mathscr{V}}$ are all bijective and commutative. The mappings (17) and (18) are inclusion reversing. The mapping (19) is inclusion preserving.

Proof. Obvious from (6), $\left(19^{\prime}\right)$, Lemmas $2,5,7$ and Proposition 8. Q.E.D.

§3. Here we consider a relation between Prüfer domains and affine schemes. 
For any integral domain $A$, we denote by $\mathscr{F} A$ the set of all invertible sub $A$-modules of the field of fractions $Q A$. Then $\mathscr{F} A$ is a commutative group and satisfies the following conditions:

$$
\left\{\begin{array}{r}
\text { If } I \in \mathscr{F} A \text {, then } I \text { is a finitely generated } A \text {-module } \\
\text { and then } I \text { is a fractional ideal of } A .
\end{array}\right.
$$

Definition. An integral domain $A$ is called Prüfer, if any finitely generated non zero ideal of $A$ is invertible.

Proposition 10. Let $K$ be a field and $A$ a subring of $K$.

(i) The next four conditions are equivalent:

(a) $A$ is a Prïfer domain with quotient field $K$.

(b) for any $P \in \operatorname{Spec} A, A_{P} \in \operatorname{Zar} K$.

(c) for any $P \in m$-Spec $A, A_{P} \in \operatorname{Zar} K$.

(d) $A$ is integrally closed in $K$ and the mapping $\Phi_{K \mid A}: \operatorname{Zar}(K \mid A) \rightarrow$ Spec $A$ is an injection.

(ii) If $K$ and $A$ satisfy one of above conditions, then $\left(\Phi_{K \mid A}, \Phi_{K \mid}\right)$ is an isomorphism of locally ringed spaces. Hence, $\operatorname{Zar}(K \mid A)$ is an affine scheme. $\Phi_{K \mid A}$ is an inclusion-reversing homeomorphism and the mapping: $P \mapsto A_{P}$ is the inverse map of $\Phi_{K \mid A}$.

Proof. It is sufficient to prove that (d) implies (b). Note that $\Phi_{K \mid A}^{-1}(P)=\Phi_{K \mid A}^{-1}\left(P_{P}\right)$ for any $P \in \operatorname{Spec} A$. Since $A_{P}$ is integrally closed in $K$, by Lemma 5 (ii), we have $A_{P}=\cap_{R \in \Phi_{K \mid A}^{-1}(P)} R$. Since $\Phi_{K \mid A}$ is injective, we have $A_{P}=R \in \operatorname{Zar} K$. For the other part of proof, see [4], Theorem 64 and Theorem 65.

Q.E.D.

Proposition 11. Let $K$ be a field, $X$ a non empty subset of Zar $K$ and $\left(X, \mathscr{O}_{X}\right)$ an affine scheme. Then $\mathcal{O}_{X}(X)$ is a Prüfer domain with quotient field $K$, and $X=\operatorname{Zar}\left(K \mid \mathcal{O}_{X}(X)\right)$.

Proof. If we put $A=\mathscr{O}_{X}(X)$, then by the assumption there exists an isomorphism $(\phi, \theta):\left(X, \mathscr{O}_{X}\right) \rightarrow(\operatorname{Spec} A, \widetilde{A})$ of locally ringed spaces. Composing suitable automorphism of $\operatorname{Spec} A$, we may assume that $\theta$ (Spec A) is the identity map of $A$. For $f \in A, f \neq 0$, we put $V_{f}=\phi^{-1}(D(f))$, and for $P \in \operatorname{Spec} A$, we put $R=\phi^{-1}(P) \in X$. Then we have the ring isomorphisms $\theta(D(f)): A_{f} \rightarrow \mathcal{O}_{X}\left(V_{f}\right)$ and $\theta_{R}: A_{P} \rightarrow R$. If we denote by $Q A$ the field of fractions of $A$, then we have the ring isomorphism $\bar{\theta}: Q A \rightarrow$ $K$. Especially, if $P \in D(f)$, then we have the following commutative diagram: 


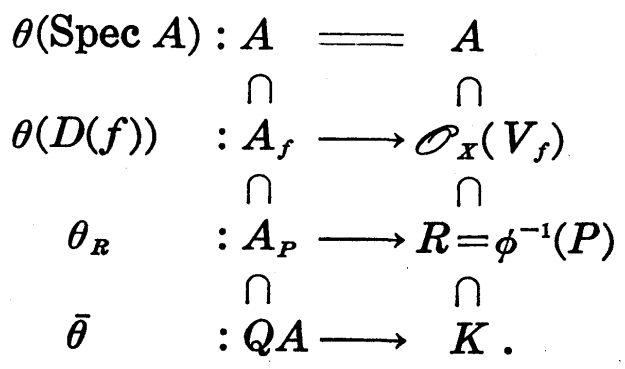

Here $\bar{\theta}$ is an $A$-isomorphism, we have $Q A=K$ and $\bar{\theta}$ is the identity map of $K$. Hence $A_{f}=\mathcal{O}_{X}\left(V_{f}\right)$ and $A_{P}=R \in \operatorname{Zar} K$. By Proposition 10, $A$ is a Pruifer domain with the field of fractions $K$. It is clear that $X \subset \operatorname{Zar}(K \mid A)$. Conversely, for any $R \in \operatorname{Zar}(K \mid A)$, we put $P=\Phi_{K \mid A}(R) \in \operatorname{Spec} A$. Since $R=A_{P}=\phi^{-1}(P) \in X$, we have $\operatorname{Zar}(K \mid A) \subset X$. Q.E.D.

Lemma 12. Let $K$ be a field, $A$ a subring of $K$ which is integrally closed in $K$ and $W$ the set of all closed points of $\operatorname{Zar}(K \mid A)$. If the restriction map of $\Phi_{K \mid A}$ to $W$ is injective, then $A$ is a Prüfer domain with quotient field $K$.

Proof. Let $P \in m$-Spec $A$ and $Y$ the set of all closed points of $\operatorname{Zar}\left(K \mid A_{P}\right)$. Then, we have $A_{P}=\mathscr{O}_{Y}(Y)$. By Lemma 7, $Y=\{R \in W$ $\left.\Phi_{K \mid A}(R)=P\right\}$ and $A_{P} \in \operatorname{Zar} K$. By Proposition 10 , we obtain that $A$ is a Prüfer domain with quotient field $K$.

Q.E.D.

Here we consider the condition:

(W-6) If $R_{1}, R_{2} \in W, m\left(R_{1}\right) \cap \cap_{R \in W} R=m\left(R_{2}\right) \cap \cap_{R \in W} R$, then $R_{1}=R_{2}$

for a subset $W$ of Zar $K$. And for a fixed field $K$, we put:

$$
\begin{aligned}
& \mathscr{Z}=\left\{X \subset \operatorname{Zar} K \mid X \neq \varnothing,\left(X, \mathscr{O}_{x}\right) \text { is an affine scheme }\right\}, \\
& \mathscr{C}=\{A \mid A \text { is a Prüfer domain with quotient field } K\}, \\
& \mathscr{W}=\{W \subset \operatorname{Zar} K \mid W \text { satisfies (W-4), (W-5) and (W-6) }\} .
\end{aligned}
$$

Then,

THEOREM 13. Let $K, \mathscr{X}, \mathscr{A}, \mathscr{W}$ be as above. Then the restriction mappings of (17), (18) and (19) between $\mathscr{X}, \mathscr{A}$ and $\mathscr{W}$ are all bijective. Instead of (18), we can also write

$$
A \longmapsto W=\left\{A_{P} \mid P \in m-\operatorname{Spec} A\right\}=\Phi_{\left.K\right|_{A} ^{-1}}^{-1}(m-\operatorname{Spec} A) .
$$

Proof. Obvious from Theorem 9, Propositions 10, 11 and Lemma 12. 
COROLlaRY. Let $X$ be a subset of Zar $K$. If $\left(X, \mathscr{O}_{X}\right)$ is a locally noetherian scheme, then $\operatorname{dim} X \leqq 1$.

REMARK. Since $\{K\}=\operatorname{Zar}(K \mid K) \stackrel{\sim}{\rightarrow}$ Spec $K$ is an affine scheme, $X=\{K\}$, $A=K, W=\{K\}$ correspond to one another by the mappings (17), (18), (19). And we can also express:

$$
\begin{aligned}
& \mathscr{W}=\{X \in \tilde{\mathscr{C}} \mid X \text { satisfies }(\mathrm{W}-6)\}, \\
& \mathscr{A}=\left\{A \in \tilde{\mathscr{A}} \mid \Phi_{\mathbf{x} \mid \mathbf{4}} \text { is injective }\right\}, \\
& \mathscr{W}=\{W \in \tilde{\mathscr{W}} \mid W \text { satisfies }(W-6)\} .
\end{aligned}
$$

Here, $\left(21^{\prime}\right),\left(22^{\prime}\right)$ follow from Proposition 10 and $\left(23^{\prime}\right)$ is obvious.

Next, we consider the noetherian case. First, we shall admit the following:

LemMa 14. Assume that a ring A satisfies the following two conditions:

(i) for any $P \in m$-Spec $A, A_{P}$ is a noetherian ring.

(ii) for any $x \in A, x \neq 0$, the set $\{P \in m$-Spec $A \mid x \in P\}$ is finite.

Then $A$ is noetherian. Conversely, if $A$ is a Dedekind domain, then $A$ satisfies the above two conditions.

It is obvious that (W-1) implies (W-5). Then for a field $K$, we put:

$$
\begin{aligned}
\mathscr{Z}_{N} & =\{X \subset \operatorname{Zar} K \mid X \neq \varnothing, X \neq\{K\}, \\
& \left.\left(X, \mathscr{O}_{X}\right) \text { is a noetherian affine scheme }\right\} \\
= & \left\{X \in \mathscr{O} \mid X \neq\{K\},\left(X, \mathscr{O}_{X}\right) \text { is a noetherian scheme }\right\}, \\
\mathscr{C}^{N}= & \{A \mid A \text { is a Dedekind domain with quotient field } K\} \\
= & \{A \in \mathscr{A} \mid A \text { is noetherian and not a field }\}, \\
\mathscr{W}_{N} & =\{W \subset \operatorname{Zar} K \mid W \text { satisfies }(\mathrm{W}-1),(\mathrm{W}-2),(\mathrm{W}-4) \text { and }(\mathrm{W}-6)\} \\
& =\{W \in \mathscr{W} \mid W \text { satisfies }(\mathrm{W}-1) \text { and }(\mathrm{W}-2)\} .
\end{aligned}
$$

Then,

THEOREM 15. Let $K, \mathscr{X}_{N}, \mathscr{A}^{N}, \mathscr{W}_{N}$ be as above.

(i) The restriction mappings of (17), (18) and (19) between $\mathscr{Q}_{N}$, $\mathscr{N}^{N}$ and $\mathscr{W}_{N}$ are all bijective. Instead of (19), we can also write

$$
W \longmapsto X=W \cup\{K\}, \quad X \longmapsto W=X-\{K\} .
$$

(ii) If $X \in \mathscr{Z}_{N}, A \in \mathscr{A}^{N}$ and $W \in \mathscr{W}_{N}$ correspond to one another by 
the mappings (17), (18), and (19), then the (Weil) divisor group Div $X$ of $X$ is the free abelian group generated by $W$ (over $Z$ ) and is isomorphic to the ideal group $\mathscr{F A}$ of $A$. And the divisor class group of $X$ is isomorphic to the ideal class group of $A$.

Proof. (i) is clear by Theorem 13, Remark and Lemma 14.

(ii) Restricting the map $\Phi_{K \mid A}$, we have an inclusion-reversing homeomorphism:

$$
W \stackrel{\sim}{\longrightarrow} m-\operatorname{Spec} A \text {. }
$$

Since a prime divisor of $X$ is identified with an element of $W$, we have Div $X \stackrel{\sim}{\rightarrow} \mathscr{I} A$ by the definition of Weil divisor group and $\left(10^{\prime}\right.$ ) (See [2], condition (*) in p. 130). For $R \in W$, we denote by $\operatorname{ord}_{R}$ the normalized discrete valuation of $K$ associated with $R$. Since $a A=\prod_{R \in W}\left(\Phi_{K \mid A} R\right)^{\operatorname{ord}_{R} a}$ holds for all $a \in K^{*}$, we have the last statement.

EXAMPLES. Let $W$ be a non empty finite subset of Zar $K$. Then:

(i) $\mathscr{O}_{W}(W) \in \mathscr{A}$.

(ii) If $W$ is a $T_{1}$-space, then $W \in \mathscr{Y}$.

(iii) If $W$ satisfies (W-1), then $\mathscr{O}_{W}(W) \in \mathscr{A}^{N}$. Moreover, $\mathscr{O}_{W}(W)$ is a principal ideal domain.

(iv) If $W$ is a $T_{1}$-space and satisfies (W-1), then $W \in \mathscr{W}_{N}^{2}$.

REMARK. On conditions for a subset $W$ of $\operatorname{Zar} K$ :

(i) (W-1) implies (W-5). (W-4) implies (W-0) (already described).

(ii) If $W \in \mathscr{W}$, then $W$ satisfies (W-3).

(iii) (W-3) implies (W-5) and (W-6).

Hence, we also have,

$$
\begin{aligned}
& \mathscr{W}=\{W \subset \operatorname{Zar} K \mid W \text { satisfies }(\mathrm{W}-3) \text { and }(\mathrm{W}-4)\}, \\
& \mathscr{W}_{N}=\{W \subset \operatorname{Zar} K \mid W \text { satisfies (W-1), (W-2), (W-3) and (W-4) }\}
\end{aligned}
$$

§4. Here we consider some applications of Theorems 9,13 and 15. For this purpose, we shall assume the following four properties for Prüfer domains and Krull domains (the definition will be stated below).

Lemma 16. Let $A$ be a Prïfer domain, $K$ the quotient field of $A$ and $L$ an algebraic extension of $K$. Then the integral closure $B$ of $A$ in $L$ is a Prïfer domain with quotient field L (See [1], (22.3), p. 277).

Lemma 17. Let $A$ be an integrally closed integral domain and $B$ 
an integral extension of $A$. If $B$ is Prïfer, then $A$ is Prüfer (See [1], (22.4), p. 278).

Definition. Let $A$ be an integral domain and $K$ the quotient field of $A$. Then $A$ is called a Krull domain, if there exists $W \subset$ Zar $K$ satisfying the conditions (W-1) and (W-2) such that $A=\cap_{R \in W} R$.

REMARK. In the above definition, $W$ can be the empty set. In that case, $A=K$, that is also a Krull domain (See $\left(8^{\prime}\right)$ ).

LEMMA 18. The following three conditions for a ring $A$ are equivalent:

(a) A is a Dedekind domain or a field.

(b) $A$ is a Prïfer domain and a Krull domain.

(c) $A$ is a Krull domain and $\operatorname{dim} A \leqq 1$.

(See [1], (43.16), p. 536).

LEMMA 19. Let $A$ be a noetherian domain, $K$ the quotient field of $A$ and $L$ a finite extension of $K$. Then the integral closure $B$ of $A$ in $L$ is a Krull domain with quotient field $L$ (See [5], Theorem 8.3.5, p. 193).

Using these results, we consider some applications to number theory.

THEOREM 20. Let $A$ be a noetherian subring of a field $K$. Then,

(i) The following three conditions are equivalent:

(a) $\operatorname{Zar}(K \mid A)$ is a scheme.

(b) $\operatorname{dim} \operatorname{Zar}(K \mid A) \leqq 1$.

(c) $\operatorname{dim} A+\operatorname{tr}-\operatorname{deg}_{Q \Delta} K \leqq 1$.

(ii) The following three conditions are equivalent:

(d) $\operatorname{Zar}(K \mid A)$ is an affine scheme.

(e) $\operatorname{dim} \operatorname{Zar}(K \mid A)=\operatorname{dim} A \leqq 1$.

(f) $K$ is an algebraic extension of $Q A$ and $\operatorname{dim} A \leqq 1$.

Proof. (i) (a) implies (b). Let $U$ be any affine open set of $\operatorname{Zar}(K \mid A)$ expressed as $U=\operatorname{Zar}\left(K \mid A_{0}\right), A_{0}=A\left[x_{1}, \cdots, x_{n}\right]$ for some $x_{1}, \cdots, x_{n} \in K$. Let $A_{1}$ be the integral closure of $A_{0}$ in $Q A_{0}$ and $B$ the integral closure of $A_{0}$ in $K$. Since $U$ is affine, $B$ is a Prüfer domain with quotient field $K$. By Lemma 17, $A_{1}$ is a Prüfer domain. On the other hand, by Lemma 19, $A_{1}$ is a Krull domain. By Lemma 18 , we have $\operatorname{dim} A_{1} \leqq 1$ and $\operatorname{dim} U=$ $\operatorname{dim} B=\operatorname{dim} A_{1} \leqq 1$. Hence we obtain that $\operatorname{dim} \operatorname{Zar}(K \mid A) \leqq 1$.

(b) implies (c). This is obvious from Lemma 6, (ii).

(c) implies (a). If $\operatorname{tr}-\operatorname{deg}_{Q A} K=0$, then $\operatorname{dim} A \leqq 1$. Let $A_{1}$ be the integral 
closure of $A$ in $Q A$ and $B$ the integral closure of $A$ in $K$. By Lemma 19, $A_{1}$ is Krull and $\operatorname{dim} A_{1} \leqq 1$. By Lemma 18, $A_{1}$ is Prüfer. And by Lemma $16, B$ is Prüfer and $K=Q B$. Hence $\operatorname{Zar}(K \mid A)=\operatorname{Zar}(K \mid B) \simeq \operatorname{Spec} B$ is an affine scheme. If $\operatorname{tr}-\operatorname{deg}_{Q A} K=1$, then $\operatorname{dim} A=0$. There exists an element $x \in K$ such that $x$ is transcendental over $A$. Then $K$ is an algebraic extension over $A(x)$. Let $A_{1}, A_{2}$ be the integral closure of $A[x], A\left[x^{-1}\right]$ in $K$, respectively. Then $A_{1}$ and $A_{2}$ are Prüfer domains with quotient field $K$. And we obtain an open covering $\operatorname{Zar}(K \mid A)=$ $\operatorname{Zar}\left(K \mid A_{1}\right) \cup \operatorname{Zar}\left(K \mid A_{2}\right)$. Since $\operatorname{Zar}\left(K \mid A_{i}\right) \simeq \operatorname{Spec} A_{i}(i=1,2)$ is an affine scheme, $\operatorname{Zar}(K \mid A)$ is a scheme.

(ii) It is sufficient to prove that if $\operatorname{dim} A=0$ and $\operatorname{tr}-\operatorname{deg}_{Q A} K=1$, then $\operatorname{Zar}(K \mid A)$ is not affine. Let $X=\operatorname{Zar}(K \mid A)$. Then $\mathcal{O}_{X}(X)$ is a field. Since $\operatorname{dim} X=1, X$ is not affine. The other parts of proof are similar to (i).

Q.E.D.

Corollary. Let $X$ be an open subset of Zar $K$ and $\left(X, \mathcal{O}_{X}\right)$ a scheme. Then $\operatorname{dim} X \leqq 1$.

Letting $A$ be the prime integral domain of $K$ in Theorem 20, we have,

THEOREM 21. Let $K$ be a field. Then:

(i) Zar $K$ is a scheme if and only if $K$ is an algebrac extension over a prime field or an extension over a finite field of transcendental degree one.

(ii) Zar $K$ is an affine scheme if and only if $K$ is an algebraic extension over a prime field.

Suppose that Zar $K$ is a scheme of finite type over $Z$. Then $K$ is a finitely generated field over the prime field. Hence we have:

THeOREM 22. Let $K$ be a field. Then, Zar $K$ is a scheme of finite type over $Z$, if and only if $K$ is a finite field or a global field of dimension one.

Letting $A$ be a subfield of $K$ in Theorem 20, we have:

TheOREm 23. Let $C$ be a subfield of a field $K$.

(i) $\operatorname{Zar}(K \mid C)$ is a scheme if and only if $\operatorname{tr}-\operatorname{deg}_{C} K \leqq 1$. In this case, the equality $\operatorname{dim} \operatorname{Zar}(K \mid C)=\operatorname{tr}-\operatorname{deg}_{C} K$ holds.

(ii) The next four conditions are equivalent:

(a) $\operatorname{Zar}(K \mid C)$ is an affine scheme.

(b) $\{K\}$ is an open subset of $\operatorname{Zar}(K \mid C)$. 
(c) $K$ is an algebraic extension over $C$.

(d) $\operatorname{dim} \operatorname{Zar}(K \mid C)=0$.

Proof. By Hilbert's Nullstellensatz (weak form), we obtain that (b) implies (c). The other parts of proof follow from Lemma 6 and Theorem 20.

Q.E.D.

To consider the similar formula to (b) over an integral domain, we introduce the notion of $G$-domains.

DEFinition. An integral domain $A$ is called a $G$-domain, if there exist $x_{1}, x_{2}, \cdots, x_{n} \in Q A$ such that $Q A=A\left[x_{1}, x_{2}, \cdots, x_{n}\right]$ (see [4], p. 12).

Then:

Proposition 24. Let $A$ be a subring of a field $K$ which is integrally closed in $K$. Then the following two conditions are equivalent:

(a) $A$ is a G-domain with quotient field $K$.

(b) $\{K\}$ is an open subset of $\operatorname{Zar}(K \mid A)$.

Proof. It is obvious that (a) implies (b).

(b) implies (a). We can express as $\{K\}=\operatorname{Zar}\left(K \mid A\left[x_{1}, \cdots, x_{n}\right]\right)=$ $\operatorname{Zar}\left(K \mid Q A\left[x_{1}, \cdots, x_{n}\right]\right)$ for some $x_{1}, \cdots, x_{n} \in K$. By Lemma 6, $Q A \subset$ $A\left[x_{1}, \cdots, x_{n}\right] \subset K$. By Theorem $23, K$ is an algebraic extension of $Q A$. Hence we have $K=Q A$.

Q.E.D.

Next we consider some applications to algebraic geometry.

Let $C$ be any field, $\left(V, \mathscr{O}_{V}\right)$ a proper integral scheme over $C$ and $K=$ Rat $V$ the function field of $V$. Then the mapping $\Phi_{V}: \operatorname{Zar}(K \mid C) \rightarrow V$ is defined by

$$
\Phi_{V}(R)=x \text { if and only if } R \text { dominates } \mathscr{O}_{V, x}
$$

for $R \in \operatorname{Zar}(K \mid C)$ and $x \in V$. Here we call $x$ the center of $R$ (see [2], p. 106). Let $U$ be an affine open set of $V$ and $A=\mathscr{O}_{V}(U)$. Then we have $Q A=K$ and

$$
\begin{aligned}
\Phi_{V}^{-1}(U)=\operatorname{Zar}(K \mid A), & \\
\operatorname{Zar}(K \mid C) \underset{\Phi_{V}}{\circlearrowright} & \longrightarrow V \\
\operatorname{Zar}(K \mid A) \underset{\Phi_{K \mid A}}{\longrightarrow} \operatorname{Spec} A & \simeq U .
\end{aligned}
$$

Let $Z=\operatorname{Zar}(K \mid C)$. Then the morphism $\Phi_{V}^{*}: \mathcal{O}_{V} \rightarrow\left(\Phi_{V}\right)_{*} \mathcal{O}_{Z}$ of sheaves 
of rings on $V$ is defined by the similar formula to (11). And the induced mapping of $\Phi_{V}^{\#}$ on the stalks is denoted by

$$
\left(\Phi_{V}^{*}\right)_{R}: \mathscr{O}_{V, x} \subset R
$$

for $R \in Z$ and $x=\Phi_{V}(R) \in V$. Hence we obtain that $\left(\Phi_{V}, \Phi_{V}^{*}\right)$ is a morphism of locally ringed spaces and

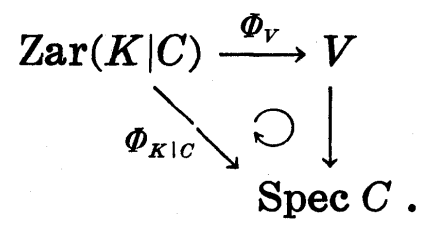

The locally ringed space $Z=\operatorname{Zar}(K \mid C)$ is clearly a birational invariant of $V . \quad \Phi_{V}$ is surjective and maps the closed points of $Z$ to that of $V$. Moreover, the restriction mapping of $\Phi_{V}$ to the sets of all closed points is also surjective. And, we have

$V$ is normal if and only if $\Phi_{V}^{*}$ is an isomorphism .

If $\Phi_{V}$ is injective, then $\operatorname{dim} V \leqq 1$.

$\operatorname{dim} V \leqq 1$ if and only if $Z$ is a scheme.

$\left(\Phi_{V}, \Phi_{V}^{*}\right)$ is an isomorphism if and only if $\operatorname{dim} V \leqq 1$ and $V$ is non-singular .

Especially, if $\operatorname{dim} V=1$, then $V$ is a projective algebraic curve and the diagram (31) is the normalization and the non-singularization (that is, the resolution of singulalities). Here we consider the case of dimension one. From Theorems 15 and 23, we have:

TheORem 25. Let $C$ be a subfield of a field $K, Z=\operatorname{Zar}(K \mid C)$ and $Y=Z-\{K\}$. Then $K$ is an algebraic function field of one variable with coefficient field $C$ if and only if $Z$ is a scheme of finite type over $C$ and $C=\mathcal{O}_{Z}(Z) \neq K$. In this case, $Z, C$ and $Y$ correspond to one another by the mappings (17), (18), (19) and $\operatorname{Div} Z=\bigoplus_{R \in Y} Z R$ holds.

In what follows, we always denote by $K$ an algebraic function field of one variable with the coefficient field $C$. Further, let $Z=\operatorname{Zar}(K \mid C)$ and $Y=Z-\{K\}$.

Lemma 26. (i) $Y$ satisfies (W-0), (W-1), (W-2), (W-4) and (W-5) but does not satisfy (W-3) and (W-6).

(ii) If $A \in \tilde{\mathscr{A}}, C \varsubsetneqq A \varsubsetneqq K$, then $A \in \mathscr{A}^{N}$. 
Proof. (i) is well-known. (ii) follows from Lemma $18 . \quad$ Q.E.D.

Lemma 27. (i) If $X \in \tilde{\mathscr{P}}$ and $W \in \tilde{\mathscr{W}}$ correspond to each other by the mappings (19), then $\{K\} \varsubsetneqq X \subset Z$ if and only if $W \subset Y$. In this case, $X$ and $W$ also correspond to each other by the mappings $\left(19^{\prime \prime}\right)$.

(ii) If $K \in X \subset Z$, then $X \in \tilde{\mathscr{Z}}$. And if $\varnothing \neq W \subset Y$, then $W \in \tilde{\mathscr{W}}$.

(iii) If $\{K\} \varsubsetneqq X \varsubsetneqq Z$, then $X \in \mathscr{Z}_{N}$. And if $\varnothing \neq W \varsubsetneqq Y$, then $W \in \mathscr{W}_{N}$.

(iv) For a subset $X$ of $Z, X$ is a non empty open subset of $Z$ if and only if $Z-X$ is finite and $K \in X$. For a subset $W$ of $Y, W$ is a non empty open subset of $Y$ if and only if $Y-W$ is finite.

Proof. (i) is obvious.

(ii) Let $x(R)$ be an element of $K-C$ which has a pole only at $R$, for any $R \in Y$. Then we have

$$
\begin{aligned}
X & =Z \cap \underset{R \in Z-X}{\cap} \operatorname{Zar}(K \mid\{x(R)\}) \\
& =\operatorname{Zar}(K \mid C \cup\{x(R) \mid R \in Z-X\}) .
\end{aligned}
$$

Hence $X \in \tilde{\mathscr{Z}}$. For a non empty subset $W$ of $Y$, we put $X=W \cup\{K\}$. Then $X \in \tilde{\mathscr{Z}}$. Hence $W=X-\{K\} \in \tilde{\mathscr{W}}$ by (i).

(iii) Let $A=\mathcal{O}_{X}(X)$. Then $C \varsubsetneqq A \varsubsetneqq K$. Hence $A \in \mathscr{A}^{N}$ by Lemma 26. Thus we obtain $X=\operatorname{Zar}(K \mid A) \in \mathscr{Z}_{N}$. For a subset $W$ of $Y$, the proof is similar to (ii).

(iv) is obvious.

Q.E.D.

Hence we obtain,

THEOREM 28. Let $K, C, Z$ and $Y$ as above. Then:

(i) The restriction mappings of (17), (18) and (19") between the set of all $X$ satisfying $\{K\} \varsubsetneqq X \varsubsetneqq Z$, the set of all $A \in \mathscr{A}^{N}$ satisfying $C \subset A \subset K$ and the set of all $W$ satisfying $\varnothing \neq W \varsubsetneqq Y$ are all bijective.

(ii) Let $X$ be a non empty open subset of $Z$ and $X \neq Z$. Then $X$ is an affine open subset of $Z$.

(iii) Let $X, A$ and $W$ correspond to one another by the mappings (17), (18), (19") in (i). Then the following three conditions are equivalent: (a) $X$ is open in $Z$.

(b) $A$ is a finitely generated ring over $C$.

(c) $W$ is open in $Y$.

In this case, we can also denote $A=\mathscr{O}_{Z}(X)=\mathscr{O}_{Y}(W), X=\operatorname{Zar}(K \mid A) \simeq$ Spec $A$ and $W \simeq m-\operatorname{Spec} A$.

Proof. (i), (ii) are obvious. 
(iii) It is clear that (a) and (c) are equivalent. It is also clear that (b) implies (a). (a) implies (b). Let $X$ be an open subset of $Z$. Then $Z-X$ is a finite set by Lemma 27, (iv). And by (36), we obtain that $A$ is a finitely generated ring over $C$. Q.E.D.

\section{References}

[1] R. Gilmer, Multiplicative Ideal Theory, Marcel Dekker (1972).

[2] R. HARTSHORne, Algebraic Geometry, Springer-Verlag (1977).

[3] S. Iyanaga (ed.), The Theory of Numbers, North-Holland (1975).

[4] I. Kaplansky, Commutative Rings, Allyn and Bacon (1970).

[5] M. NAGATA, Kakankanron, Kinokuniya (1974), (in Japanese).

Present Address:

Department of Mathematics, Sophia University

KIOIChO, ChIYOdA-KU, TOKYo 102, JAPAN 